\title{
Modelo de evaluación del bienestar estudiantil universitario en Colombia
}

\author{
Guillermo Murillo-Vargas, Carlos H. González-Campo* y Alejandro S. Piñeros \\ Universidad del Valle, Sede San Fernando, Cali-Colombia. (correo-e: guillermo.murillo@correounivalle.edu.co; \\ carlosh.gonzalez@correounivalle.edu.co; steven.pineros@correounivalle.edu.co) \\ * Autor a quien debe ser dirigida la correspondencia
}

Recibido Jul. 24, 2020; Aceptado Sep. 28, 2020; Versión final Nov. 21, 2020, Publicado Abr. 2021

\begin{abstract}
Resumen
En este artículo se propone un modelo de evaluación del bienestar estudiantil universitario que mejore la calidad de vida de la comunidad estudiantil en las instituciones de educación superior en Colombia. La investigación fue de carácter cualitativo a través de la revisión documental y de talleres con expertos en bienestar y en evaluación, con una revisión de la literatura previa de diferentes modelos de evaluación en varios países, con distintos enfoques y desde varias disciplinas. Lo anterior, permitió la identificación de los componentes a evaluar, partiendo de la identificación de los servicios comunes en los modelos de bienestar existentes en las universidades acreditadas con el mayor número de años, para finalmente, proponer la formulación del modelo. Se concluye que el modelo brinda la oportunidad de hacer seguimiento a los diferentes programas implementados en el marco del bienestar universitario, a propósito, del mejoramiento continuo definido en la actualización normatividad de 2019 y 2020 en Colombia.
\end{abstract}

Palabras clave: modelo; evaluación; bienestar institucional; gestión; políticas

\section{Model for evaluation of university student well-being in Colombia}

\begin{abstract}
The present study proposes a model for evaluating the well-being of university students to improve the quality of life of the student community in higher education institutions in Colombia. The research is qualitative in nature and is performed by conducting documentary reviews and workshops with well-being and evaluation experts. A literature review is performed to examine evaluation models from other countries, disciplines, and approaches. This allowed the identification of the components examined, which included the identification of common services in welfare models from the oldest accredited universities. It is concluded that the model presented here provides the opportunity to monitor programs implemented within university well-being frameworks, which meets the continuous improvement definition in the 2019 and 2020 Colombian regulatory updates.
\end{abstract}

Keywords: evaluation model; institutional well-being; management; policies 


\section{INTRODUCCIÓN}

En Colombia el Ministerio de Educación Nacional (MEN), aprueba el otorgamiento o la renovación de los registros calificados para el funcionamiento de los programas académicos de pregrado o posgrado en las diferentes modalidades desde el cumplimiento de las 15 condiciones de calidad definidas en el Decreto 1330 de 2019. En este contexto, por medio de la Resolución 15224 de agosto 24 de 2020, es obligatorio presentar como una evidencia necesaria para el cumplimiento de la condición de calidad, el Modelo de Bienestar y la descripción de los procesos de evaluación del bienestar. Con lo anterior, se requiere que las 299 Instituciones de Educación Superior (IES) que ofrecen educación superior en el país a más de 2,4 millones de estudiantes, diseñen y estructuren metodologías que les permitan realizar la evaluación requerida. El objetivo de este artículo es responder la pregunta de investigación: ¿Cuál es modelo requerido para la evaluación del bienestar estudiantil en una Universidad en Colombia?

Según la anterior normatividad, las IES en el cumplimiento de la condición de calidad "Modelo de Bienestar", deben definir "políticas, procesos, actividades y espacios que complementan y fortalecen la vida académica y administrativa", orientadas al "desarrollo integral de la persona y la convivencia". En este marco normativo, cada institución deberá ofrecer un conjunto de servicios de bienestar a la comunidad estudiantil, de acuerdo con la naturaleza jurídica y la identidad de la institución; también debe contar con medios de comunicación y divulgación para su difusión, además de mecanismos para realizar la evaluación de los mencionados servicios.

La diversidad de las IES en Colombia hace necesario que, tanto del Modelo de Bienestar, como los mecanismos para su evaluación, respondan a esas características particulares de las instituciones, que están determinadas, tal como lo plantea el Decreto 1330 de 2019 (MEN, 2019), por la naturaleza jurídica, la identidad, el carácter, la tipología y la misión institucional. Así mismo se establece que los procesos de autoevaluación y autorregulación, que para efectos de esta investigación deben orientar entre otros a los mecanismos de evaluación del bienestar estudiantil; estén bajo los principios de equidad, diversidad, inclusión y sostenibilidad. En el modelo de acreditación de alta calidad de instituciones y programas, actualizado mediante el Acuerdo 02 de 2020 del Consejo Nacional de Educación Superior (CESU, 2020), se incorpora en el Factor Bienestar institucional, las evidencias dentro de la descripción de las características del seguimiento a los programas y las actividades realizadas con la comunidad universitaria.

El Ministerio de Educación Nacional previo a la modificación normativa realizada en el 2019 para el registro calificado de programas, y en el 2020 para los procesos de acreditación en alta calidad, publicó en el 2016, un documento donde se definieron los lineamientos de política de bienestar para instituciones de educación superior, que incluyen la aproximación al concepto de bienestar en la educación superior, los principios de las política, las características del sistema, las dimensiones y el bienestar institucional y los componentes del bienestar en las IES. El concepto de bienestar en las IES, propuesto por el MEN en el 2016, tiene dos partes, la primera como "una función de carácter estratégico y transversal que comprende las políticas institucionales, los procesos y las prácticas"; y la segunda, como "una cultura institucional que promueve la corresponsabilidad de los actores que conviven en el contexto de la vida institucional para favorecer la formación integral, el pleno desarrollo de las capacidades humanas y la construcción de comunidad". (MEN, 2016, p. 26).

Dentro de los componentes del bienestar, según el MEN (2016), está incluido el componente de evaluación, seguimiento y monitoreo, asociado a la gestión de datos e información para evidenciar los logros de las actividades de bienestar, y para identificar las acciones de mejoramiento. Así mismo, se propone la necesidad de articular estas acciones a la planeación institucional y a los proyectos de inversión o financiación. Entre los aspectos a evaluar propuestos dentro de los lineamientos se encuentran la permanencia, el clima organizacional y el desarrollo de los egresados; en lo establecido por el Ministerio de Educación Nacional se plantea la necesidad de utilizar encuestas de percepción y satisfacción.

Teniendo en cuenta lo anterior, esta investigación se propone como una metodología para contestar la pregunta de investigación, y que las IES cuenten con un referente en el contexto colombiano que les permita atender los nuevos requerimientos normativos definidos en el 2019 y 2020, los siguientes pasos: En la primera fase, una revisión de los modelos de bienestar de las 10 Instituciones de Educación Superior acreditadas institucionalmente con 8 años y las 5 instituciones acreditadas con 10 años, máximo reconocimiento en años que realiza el MEN. En la segunda fase, para establecer las dimensiones del modelo de bienestar y los aspectos a evaluar en cada dimensión, se realizan talleres con expertos de las diferentes áreas de bienestar, y con expertos en evaluación desde diferentes áreas de conocimiento. Finalmente, se construye el modelo de evaluación del bienestar estudiantil universitario, la utilización del modelo para la evaluación del bienestar estudiantil en una universidad no es una fase, ni es el alcance de esta investigación. Previamente, en la revisión de la literatura se examinaron modelos de evaluación del bienestar estudiantil universitario existentes 
en otros países, desde diferentes perspectivas y con distintos enfoques, para establecer elementos que se pueden articular con el modelo propuesto, pero también para identificar las diferencias en la discusión.

\section{REVISIÓN DE LITERATURA}

Para responder la pregunta de investigación propuesta, se realizó una revisión literaria de modelos de evaluación del bienestar en diferentes universidades, partiendo de búsquedas realizadas en Web of Science (WoS) con la ecuación de busquedad welfare* OR well-being* AND university* OR "higher education*" OR college* AND Evaluation* OR Examine*, aparece un total de 235, en la revisión del contenido con relación a la pregunta de investigación y por la relevancia, en la Tabla 1, se presentan los artículos referentes para la construcción del modelo.

Tabla 1. Literatura referente desde WoS

\begin{tabular}{|l|l|l|l|l|l|}
\hline \multicolumn{1}{|c|}{ Articulo } & \multicolumn{1}{|c|}{$\begin{array}{c}\text { Tipo de } \\
\text { Investigación }\end{array}$} & \multicolumn{1}{|c|}{ Sujetos de estudio } & $\begin{array}{c}\text { País } \\
\text { investigación }\end{array}$ & $\begin{array}{l}\text { Cantidad de } \\
\text { sujetos }\end{array}$ & \multicolumn{1}{c|}{ Categoría } \\
\hline $\begin{array}{l}\text { Campos- } \\
\text { Uscanga et al. } \\
(2016)\end{array}$ & Cuantitativa & $\begin{array}{l}\text { Estudiantes } \\
\text { universitarios }\end{array}$ & México & 424 & Psicología \\
\hline $\begin{array}{l}\text { Baldwin et al. } \\
(2017)\end{array}$ & Cuantitativa & $\begin{array}{l}\text { Estudiantes de } \\
\text { pregrado }\end{array}$ & Estados Unidos & 211 & Psicología Clínica \\
\hline $\begin{array}{l}\text { Morris-Paxton et } \\
\text { al. (2017) }\end{array}$ & Mixto & $\begin{array}{l}\text { Estudiantes de } \\
\text { pregrado }\end{array}$ & Sudáfrica & 34 & $\begin{array}{l}\text { Salud pública, Salud } \\
\text { ambiental y } \\
\text { ocupacional }\end{array}$ \\
\hline $\begin{array}{l}\text { Mayol et al. } \\
(2017)\end{array}$ & Cuantitativa & $\begin{array}{l}\text { Estudiantes } \\
\text { universitarios atletas } \\
\text { y no atletas }\end{array}$ & Estados Unidos & 1699 & $\begin{array}{l}\text { Salud pública, } \\
\text { ambiental y } \\
\text { ocupacional }\end{array}$ \\
\hline $\begin{array}{l}\text { McNeilly y } \\
\text { Raming (2018) }\end{array}$ & Cuantitativa & $\begin{array}{l}\text { Estudiantes } \\
\text { universitarios }\end{array}$ & Estados Unidos & 86 & $\begin{array}{l}\text { Salud Pública, Medio } \\
\text { Ambiente y Salud } \\
\text { Ocupacional }\end{array}$ \\
\hline
\end{tabular}

En Scopus la ecuación de busquedad fue (TITLE-ABS-KEY ( welfare* OR well-being ${ }^{*}$ ) AND TITLE-ABS-KEY (university* OR "higher education*" OR college*)), dando un total de 14893 documentos ordenados por relevancia, de los cuales se exploraron los primeros 100, y por la relación con el estudio se seleccionaron los que se presentan en la Tabla 2.

Tabla 2. Literatura referente desde Scopus

\begin{tabular}{|l|l|l|l|l|l|}
\hline \multicolumn{1}{|c|}{ Articulo } & \multicolumn{1}{c|}{$\begin{array}{c}\text { Tipo de } \\
\text { Investigación }\end{array}$} & \multicolumn{1}{|c|}{ Sujetos de estudio } & $\begin{array}{c}\text { País } \\
\text { investigación }\end{array}$ & $\begin{array}{l}\text { Cantidad } \\
\text { de sujetos }\end{array}$ & \multicolumn{1}{c|}{ Categoría } \\
\hline Bahrami (2016) & Cuantitativa & $\begin{array}{l}\text { Estudiantes } \\
\text { universitarios }\end{array}$ & Irán & 310 & Ciencias Humanas \\
\hline $\begin{array}{l}\text { Salcedo et al. } \\
(2017)\end{array}$ & Cuantitativa & $\begin{array}{l}\text { Estudiantes - } \\
\text { 380 Universidad 1 } \\
226 \text { Universidad 2 }\end{array}$ & Ecuador & 606 & $\begin{array}{l}\text { Ciencias de la gestión e } \\
\text { investigación operativa }\end{array}$ \\
\hline $\begin{array}{l}\text { Figueira et al. } \\
(2017)\end{array}$ & Cuantitativa & $\begin{array}{l}\text { Estudiantes } \\
\text { universitarios }\end{array}$ & Portugal & 128 & Psicología \\
\hline Walker (2019) & Cualitativa & $\begin{array}{l}\text { Estudiantes } \\
\text { universitarios }\end{array}$ & Sudáfrica & 65 & Social Sciences \\
\hline $\begin{array}{l}\text { lordache-Platis } \\
(2020)\end{array}$ & Cualitativa & $\begin{array}{l}\text { Estudiantes } \\
\text { universitarios }\end{array}$ & Rumania & 382 & $\begin{array}{l}\text { Gestión, seguimiento, } \\
\text { política y derecho }\end{array}$ \\
\hline
\end{tabular}

En general los estudios revisados abordan modelos de evaluación del bienestar universitario que incorporan temas de nutrición, salud mental y física, temas emocionales, etc., que incidan positivamente en el estudiante y su desarrollo integral. También existen otros estudios que abordan la relación entre el bienestar académico de los estudiantes y la percepción del ambiente educacional (Hinrichs et al., 2016).

Entre los estudios referentes se encuentra Baldwin et al. (2017), donde los autores, realizaron una evaluación de aspectos del bienestar físico, emocional, social, intelectual y ocupacional, en dos universidades de Estados Unidos, para además de analizar el impacto en el rendimiento académico de los estudiantes, examinar algunas diferencias asociadas a determinantes socioeconómicos. También en Estados Unidos, y con un enfoque cualitativo de evaluación del bienestar del estudiantado, Mayol et al. (2017) y McNeilly y Raming (2018), presentan en ambos casos, evaluaciones con una orientación desde la Salud Publica. En el caso del primero, por medio de una prueba de la estructura factorial del Inventario de Bienestar Multidimensional, mientras que el segundo, mediante la encuesta Qualtrics que incluye aspectos asociados a la demografía, la seguridad alimentaria y las regulaciones del servicio de alimentos. 
En la Tabla 1 y la Tabla 2, se evidencian otros estudios sobre evaluación del bienestar de los estudiantes con enfoque cuantitativo, con la utilización de encuestas de percepción sobre condiciones, servicios y actividades, en Ecuador, Irán, México o Portugal, desde áreas como la psicología, las ciencias humanas o la investigación de operaciones. En todos los casos, los estudios incluyen elementos del contexto propio del sistema de educación superior de cada país, y de las condiciones socioeconómicas que determinan el éxito académico.

Con enfoque cualitativo, por su parte, se encuentran los estudios de Walker (2019) y lordache-Platis (2020), el primero, realizado en Sudáfrica, con una orientación desde las ciencias sociales, evaluando la relevancia del bienestar multidimensional de los estudiantes, analizando los recursos y las capacidades como un determinante. En el segundo estudio, la autora evalúa en Rumania, los servicios de bienestar y la estrategia institucional, con alcances en el contexto de la pandemia. Así mismo se identificaron estudios con enfoques mixtos, cuantitativo y cualitativo, como el propuesto por Morris-Paxton et al. (2017), en Sudáfrica, que incluye la evaluación de aspectos de salud y de prioridades sanitarias.

Entre los modelos existentes para la evaluación del bienestar estudiantil universitario, el propuesto en Motefakker (2016), es relevante para la investigación, ya que estudia el nivel de satisfacción de los estudiantes de una universidad iraní con los servicios de bienestar, en la búsqueda de mejorar la calidad de los servicios de asistencia social. En función de lo anterior, los autores diseñaron un cuestionario de evaluación con 11 variables en forma de pregunta, que, entre otras cosas, correspondieran al nivel de satisfacción con la calidad del autoservicio de comida; nivel de satisfacción con los servicios de transformación en la ciudad de Qazvin; el nivel de satisfacción con el internet universitario; el nivel de satisfacción con el sitio de la Facultad; el nivel de satisfacción con las condiciones físicas de las clases; el nivel de satisfacción con las condiciones de los dormitorios; el nivel de satisfacción con la variedad de material bibliográfico de la biblioteca; el nivel de satisfacción de las actividades y ambiente religiosos; y el nivel de satisfacción con las actividades del comité científico y los centros culturales de la universidad.

Otro modelo revisado, que incluye aspectos diferentes a los anteriores, es el propuesto por Lugosi en 2019, que explora el potencial entre el servicio de alimentación en los campus universitarios y el bienestar de los estudiantes. Este potencial se configura en términos de la provisión de comida saludable y de calidad, el apoyo en elecciones alimenticias pertinentes y la promoción de interacciones sociales equilibradas. Al respecto, se analiza que el servicio de alimentación dentro del campus ha sido reconocido como un factor sustancial que contribuye positivamente en la experiencia general del estudiante.

Finalmente, teniendo en cuenta el método de evaluación, el mayor número de aspectos evaluados y la relación con el éxito académico, se revisa el modelo propuesto en Goodday et al., (2019), donde los autores examinan la contribución de los factores de riesgo y resistencia a los resultados de bienestar y éxito académico de los estudiantes de primer año, quienes ingresan a las escuelas profesionales de negocios, medicina y derecho en la Universidad de Queen, Canadá. La encuesta de línea base incluyó cinco dominios compuestos por: factores personales, factores familiares, factores ambientales, salud emocional y psicológica y comportamientos, hábitos y estilos de vida. Por su parte, la búsqueda de ayuda de los estudiantes y el uso de servicios universitarios de salud y bienestar, fueron medidos a través de lista proporcionada por los estudiantes acerca de los servicios a los cuales habían accedido, además de las barreras percibidas en la atención. Asimismo, se les ofreció a los estudiantes la oportunidad de ampliar su opinión sobre la utilidad de los servicios y posibles sugerencias respecto a los servicios adicionales que deberían ser prestados por parte de la División de Asuntos estudiantiles de la Universidad.

La revisión de literatura permitió comparar modelos de evaluación de bienestar estudiantil universitario en diferentes países con enfoques distintos y desde diversas disciplinas y perspectivas de análisis, esta comparación sirvió como marco de referencia para la formulación del Modelo de Evaluación del Bienestar Estudiantil Universitario, el cual reúne aspectos identificados en modelos ya existentes, como la información demográfica del estudiante, los beneficios de salud, desarrollo humano y promoción socioeconómico, recreación, cultura y deporte, restaurante universitario, entre otros. A su vez, desde los hallazgos en la revisión de la literatura, en el modelo, se propone adicionalmente, la calificación y la evaluación de los medios de comunicación, su uso y calidad, además, explora las necesidades de los estudiantes y cómo los beneficios existentes pueden mejorar.

\section{METODOLOGÍA}

El diseño y el desarrollo metodológico de la investigación, está definido como un estudio de carácter cualitativo y surge a partir de cuatro fases de investigación, donde previamente a estas fases se ha realizado una revisión de la literatura en Scopus y Web of Science. Es importante precisar que la metodología en esta investigación incorpora como técnicas de recolección la entrevista semi estructurada, el análisis documental, los talleres y los grupos focales. La primera fase desarrolló un análisis comparativo de los componentes y variables de la gestión del bienestar institucional de las universidades colombianas que ostentan los mayores niveles de 
acreditación institucional que están definidos en 10 y 8 años de acreditación. Al realizar este análisis comparativo surgieron diferencias y similitudes entre las distintas universidades que permitieron construir una caracterización de los servicios ofrecidos desde el bienestar institucional universitario en Colombia. La segunda fase contempló la realización de talleres y grupos focales con los diferentes expertos de las diferentes áreas del bienestar institucional para la identificación, caracterización y agrupación de los diferentes componentes y variables del bienestar que inciden de manera significativa en los servicios que prestan las universidades al estudiantado. La tercera fase se desarrolló a partir de grupos focales y talleres con expertos externos en evaluación tanto en los aspectos cuantitativos como cualitativos de los componentes del bienestar estudiantil. La cuarta fase se genera a partir de la construcción del nuevo modelo de evaluación del bienestar estudiantil universitario, que integra las fases anteriores. En esta investigación no se incluye el proceso de implementación del modelo.

El modelo presentado en este artículo está compuesto por nueve componentes analíticos y por quince variables. El modelo de evaluación del bienestar estudiantil se construye partir de la revisión teórica y analítica, la identificación y análisis de las diferentes fases de investigación que incorpora los talleres, entrevistas y grupos focales con expertos externos en evaluación, los equipos responsables de las diferentes áreas de bienestar y la integración de los resultados del análisis comparativo con las instituciones acreditadas en Colombia por 8 y 10 años. Desde lo anterior, se agruparon las dimensiones analíticas que dieron origen a los componentes y se identificaron las diferentes variables y su correspondiente componente. Posteriormente se realizó una revisión de la propuesta del modelo a través de dos grupos focales de expertos con el propósito de validar todas las dimensiones y variables para así evitar la ausencia de alguna variable de gran importancia e impacto para el modelo de bienestar.

\section{RESULTADOS}

En la primera fase se realizó una comparación de los modelos de bienestar institucional de las universidades con 10 y 8 años de acreditación en Colombia, donde se encuentra que las áreas de bienestar que más se repiten son: Cultura, recreación y deportes; promoción de la salud, estrategias de alimentación, desarrollo estudiantil, apoyo estudiantil y seguridad, apoyo socioeconómico. También es importante señalar que en los modelos de bienestar institucional se cuenta con una estructura administrativa que normalmente depende de las rectorías y que fundamentalmente se encuentran ubicadas organizativamente en una división, sección o coordinación y en muy pocas instituciones el bienestar se encuentra dirigido desde una vicerrectoría.

Tabla 3. Variables del Modelo de Evaluación del Bienestar Estudiantil Universitario

\begin{tabular}{|c|c|}
\hline Componente & Variables \\
\hline $\begin{array}{l}\text { Información personal y } \\
\text { académica del estudiante } \\
\text { (IPAE) }\end{array}$ & $\begin{array}{l}\text { 1). Datos Personales (DP), 2) Información Socioeconómica (ISE), 3) Información } \\
\text { Académica (IA). }\end{array}$ \\
\hline Beneficios de salud (BS) & $\begin{array}{l}\text { 1). Conocimiento, uso y calificación del sistema de los diferentes servicios de salud } \\
\text { (CUYC), 2) Aporte al mejoramiento integral al estudiante (AMI), 3) Medición y control } \\
\text { del sistema de salud en cuanto a difusión y la calidad del servicio (MC), 4) Beneficios } \\
\text { (B) con citas médicas, exámenes de laboratorio, entre otros. }\end{array}$ \\
\hline $\begin{array}{l}\text { Beneficios en desarrollo } \\
\text { humano y promoción } \\
\text { socioeconómica (BDHPS) }\end{array}$ & $\begin{array}{l}\text { 1) Conocimiento, uso y calificación del sistema de los diferentes servicios de salud } \\
\text { (CUYC), 2) Aporte al mejoramiento integral al estudiante (AMI), 3) Medición y control } \\
\text { del sistema de salud en cuanto a difusión y la calidad del servicio (MC), 4): Beneficios } \\
\text { (B) con subsidios, becas de alimentación, exenciones de matrícula, entre otras, 5) } \\
\text { Desarrollo Humano (DH), aumento y mejoramiento de las capacidades, y la expansión } \\
\text { de una gama de oportunidades para un bienestar integral }\end{array}$ \\
\hline $\begin{array}{l}\text { Beneficios de recreación, } \\
\text { cultura y deportes (RCD) }\end{array}$ & $\begin{array}{l}\text { 1). Programas de recreación (PR), 2) Conocimiento, uso y calificación del beneficio } \\
\text { (CUC), 3): Aporte al mejoramiento integral al estudiante (AMI), 4) Medición y control del } \\
\text { sistema de salud en cuanto a difusión y la calidad del servicio (MC). }\end{array}$ \\
\hline $\begin{array}{l}\text { Beneficios del restaurante } \\
\text { universitario }(R U)\end{array}$ & $\begin{array}{l}\text { 1). Cobertura del servicio (C), 2) Conocimiento, uso y calificación del beneficio (CUC), } \\
\text { 3) Aporte al mejoramiento integral al estudiante (AMI), 4) Medición y control del sistema } \\
\text { de salud en cuanto a difusión y la calidad del servicio (MC). }\end{array}$ \\
\hline $\begin{array}{l}\text { Otros factores para tener } \\
\text { en cuenta (OF) }\end{array}$ & $\begin{array}{l}\text { 1). Permanencia Deserción Incidencia de los Beneficios, en cuanto a la permanencia } \\
\text { del estudiante en la institución (P-D), 2). Reingreso, con preguntas a los estudiantes de } \\
\text { reingreso acerca de los beneficios universitarios, 3) Estudiantes de Sedes Regionales, } \\
\text { por la diferencia en algunos beneficios (ESR), 4) Recomendaciones de Mejoramiento, } \\
\text { los estudiantes aportan recomendaciones para el mejoramiento de los módulos. }\end{array}$ \\
\hline
\end{tabular}


Partiendo de la revisión de la literatura con la comparación de modelos de evaluación existentes en otros países, con diferentes enfoques y desde distintas disciplinas, y la metodología propuesta para responder la pregunta de investigación, ¿Cuál es modelo requerido para la evaluación del bienestar estudiantil en una Universidad?, a continuación se presenta la formulación de un modelo interpretativo que permita integrar todas las secciones que comprenden el Modelo de Evaluación del Bienestar Estudiantil Universitario, incluyendo los diferentes componentes que lo integran. Los componentes del modelo se describen a continuación.

En la Figura 1, en el Modelo de Evaluación de Bienestar Estudiantil Universitario, se incluyen los siguientes componentes: información personal y académica del estudiantado, beneficios de salud, beneficios de desarrollo humano y promoción socioeconómica, beneficios de recreación, cultura y deportes, beneficios de restaurante universitario, preguntas de permanencia - deserción, preguntas reingreso, preguntas adicionales estudiantes sedes regionales, y el componente de preguntas recomendaciones de mejoramiento.

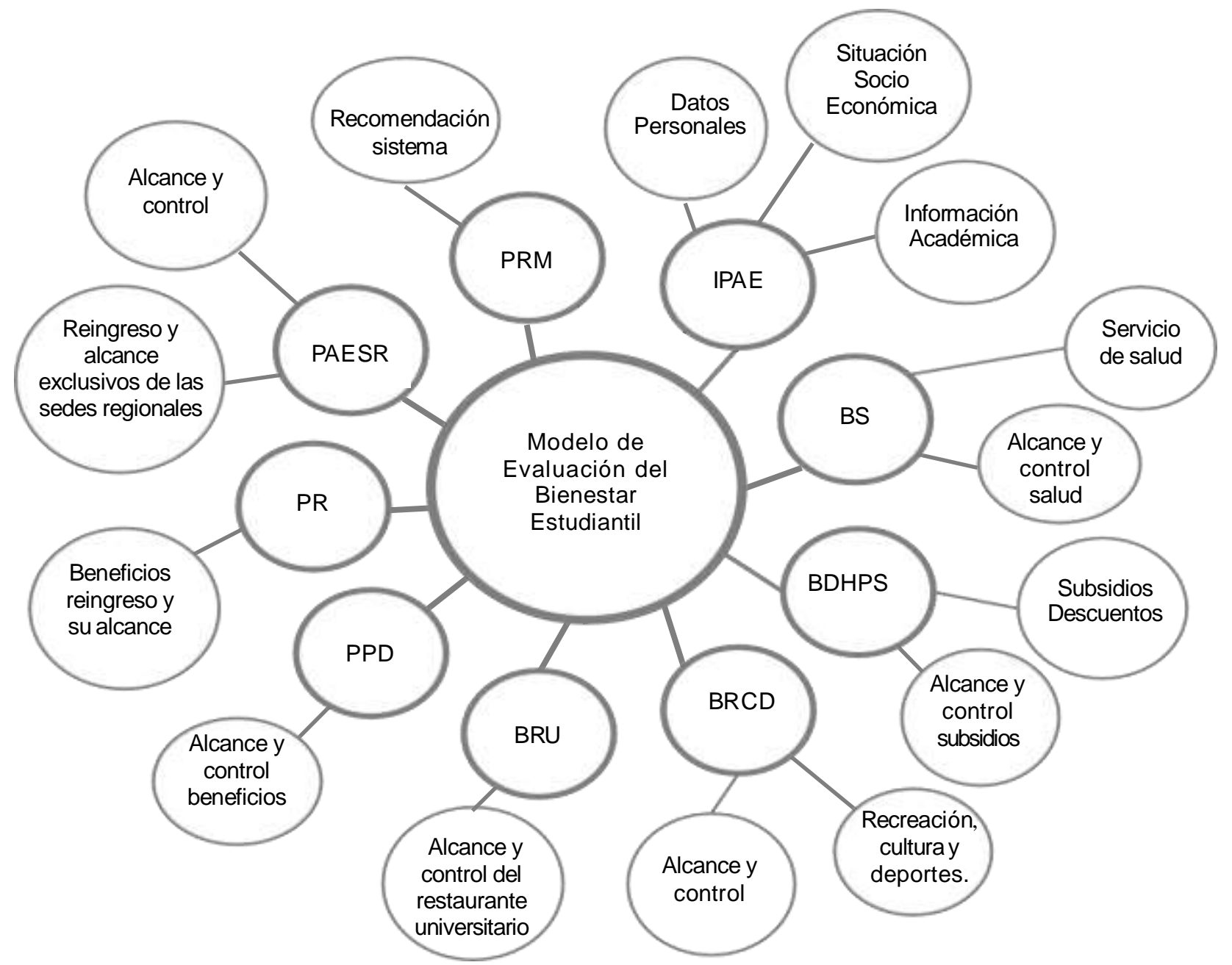

Fig 1. Componentes del Modelo de Evaluación de Bienestar Estudiantil Universitario

La propuesta interpretativa presenta las valoraciones que componen el Modelo de Evaluación del Bienestar Estudiantil, cada una de ellas tiene diferentes categorías que se interrelacionan y que permiten explicar la composición del presente modelo. La Figura 1, comprende una serie de círculos compuesta de elementos teóricos evaluativos que permiten analizar los resultados del modelo. Dado lo anterior, se articulan cada una de las diferentes valoraciones o percepciones propuestas para analizar el bienestar del estudiantado en el contexto de la educación superior en Colombia.

La aplicación del modelo propuesto se debe realizar por medio de una encuesta al estudiantado para desde su percepción realizar la evaluación de cada componente, que serán incluidos en el cuestionario para la recolección de datos, que luego serán analizados con un enfoque cuantitativo. Así mismo, en la metodología de aplicación se propone la posibilidad para por medio de entrevistas a los representantes y líderes estudiantiles, analizar los resultados de cada componente, para completar la evaluación, ahora con un enfoque cualitativo, para finalmente, proponer acciones de mejora. 
La evaluación del bienestar estudiantil constituye uno de los aspectos más destacados en los procesos de autoevaluación de las IES, en especial, con la nueva normativa definida por el Ministerio de Educación Nacional, permite identificar las diferentes necesidades a cubrir en estas comunidades y poder tomar las decisiones de carácter estratégico, que permita generar nuevos equilibrios en la asimetría de la información y nuevas formas conocimiento sobre los diferentes actores en la vida universitaria. El bienestar universitario se podrá ver fortalecido si cada vez podemos medir su impacto en el desarrollo y formación de los estudiantes. Las anteriores razones determinan la importancia del modelo propuesto.

\section{DISCUSIÓN}

Aunque como fue evidente en la revisión de la literatura, existen diferentes modelos para evaluar el bienestar universitario desde enfoques cualitativos, cuantitativos y mixtos, desde diferentes disciplinas o con distintos alcances como Goodday et al. (2019), Lugosi (2019) o Motefakker (2016), que podrían ser una opción para evaluar el bienestar estudiantil en una IES en Colombia, los limites evidenciados en cada uno, precisamente desde el enfoque, la disciplina o el alcance, hacen necesario que en el cumplimiento de lo definido en el Decreto 1330 de 2019, se proponga la necesidad de formular un Modelo de Evaluación del Bienestar Estudiantil, propio para el contexto Colombiano, y que responda la diversidad, el carácter y la naturaleza jurídica de cada institución.

Al incluir componentes ya evaluados en modelos existentes, como los temas de la salud para los estudiantes universitarios y su importancia desde la salud pública, presentes en publicaciones como Morris-Paxton et al. (2017), Mayol et al. (2017) y McNeilly y Raming (2018), se facilita la identificación de variables y escalas que fortalecen el nuevo modelo formulado. Lo mismo ocurre con componentes como los servicios de alimentación, aspectos evaluados en estudios como Lugosi (2019) y que en el modelo propuesto se encuentran dentro de los Beneficios de Restaurante Universitario (RU).

En el componente Beneficios de Desarrollo Humano y Promoción Socioeconómica (BDHPS), a diferencia de lo propuesto por Figueira et al. (2017), Campos-Uscanga et al. (2016) o Baldwin et al. (2017), estudios que evalúan los apoyos psicológicos de los estudiantes ofrecidos por las universidades y su impacto, se complementa con una visión del trabajo social, para abordar incluso temas de promoción socioeconómica, muy importantes en las diferentes regiones, subregiones y en la ruralidad existente en Colombia, con condiciones sociales y económicas que determinan el ingreso a la educación superior, y el éxito académico.

En contraste de los dos modelos evidenciados en la revisión de literatura, con el mayor número de variables evaluadas Goodday et al. (2019) y Motefakker (2016), el modelo propuesto, incluye componentes asociados a la permanencia y la deserción, el proceso de reingreso, y para el caso de IES con varias sedes o varios campus, variables asociadas a servicios diferenciales en otras sedes y un componente con variables asociadas al mejoramiento.

Es importante recalcar que en su implementación el Modelo de Evaluación de Bienestar Estudiantil Universitario, hará parte de los procesos de autoevaluación, con un enfoque mixto, donde primero, por medio del cuestionario se recolectara la información de los estudiantes, y luego, por medio de entrevistas con los representantes y líderes estudiantiles, se revisaran los resultados para encontrar algunas explicaciones complementarias, y para la definición de las acciones de mejora.

El modelo de evaluación del bienestar estudiantil configura un importante referente del rol que cumplen las universidades en la vida social, psicoafectiva y económica en las comunidades que impactan a través de los diferentes servicios ofrecidos en las unidades responsables del bienestar. También el modelo emerge como una importante estrategia para los líderes institucionales que pretenden alcanzar los objetivos trazados y ver implementados los resultados de la gestión universitaria.

Finalmente, tanto los modelos como los mecanismos de evaluación del bienestar estudiantil universitario buscan lograr un objetivo común, brindar las herramientas necesarias para impulsar el logro del éxito académico y evitar la deserción o situaciones lamentables. En ese sentido, el Modelo de Evaluación de Bienestar Estudiantil es una oportunidad de hacer seguimiento a los diferentes beneficios implementados en el marco del bienestar universitario, también explora opciones de mejora, tanto en calidad como en su difusión en las diferentes sedes. Por otro lado, es una herramienta de seguimiento que brinda información valiosa de los aspectos de bienestar universitario, para su respectiva mejora o creación de nuevos servicios que aporten al desarrollo integral de los estudiantes y disminuir indicadores como la deserción y reingresos.

\section{CONCLUSIONES}

De acuerdo al trabajo presentado y a los resultados obtenidos, se pueden plantear las siguientes conclusiones principales: (1) A partir de la literatura se identificaron las diferentes perspectivas y corrientes para la 
evaluación del bienestar estudiantil, así como las principales componentes y variables que inciden en el desarrollo de este y que permiten la evaluación a partir de un modelo que recoge las más recientes investigaciones sobre el tema y coloca de presente el rol del bienestar estudiantil en la vida universitaria; (2) El modelo propuesto de bienestar estudiantil permite un análisis integral de los aspectos de salud física y emocional, los componentes socioeconómicos, nutricionales, culturales y recreativos, entre otros, que sumados a los aspectos de formación académica permitirán orientar las políticas institucionales en materia de bienestar estudiantil en el contexto colombiano; (3) El modelo propuesto alcanza mayores niveles de integración e implementación a partir de la incorporación de las componentes y variables del bienestar estudiantil que hacen parte de las políticas institucionales de las universidades colombianas que ostentan los mayores niveles de acreditación en Colombia, 8 y 10 años de acreditación tanto en instituciones de naturaleza pública como privada;(4)El modelo de evaluación del bienestar estudiantil se convierte en un instrumento muy importante para el cumplimiento del Decreto 1330 de 2019 y del Acuerdo 02 de 2020 del Consejo Nacional de Educación Superior en Colombia (CESU).

\section{REFERENCIAS}

Baldwin, D. R., Towler, K., Oliver, M. D., y Datta, S, An examination of college student wellness: A research and liberal arts perspective. . https://doi.org/10.1177/2055102917719563 Health Psychology Open, 4(2), 205510291771956, (2017).

Bahrami, S., Relationships between spiritual well-being and quality of life in higher education, IIOAB JOURNAL, 7, 122126, (2016).

Campos-Uscanga, Y., Lagunes, R., Morales-Romero, J., y Romo-González, T, Diseño y validación de una Escala de Autorregulación de la Actividad Física. Revista de Psicología Del Deporte, 25(2), 309-316, (2016).

Figueira, C. P., Marques-Pinto, A., Pereira, C. R., y Roberto, M. S., How can Academic Context Variables Contribute to the Personal Well-Being of Higher Education Students?, https://doi.org/10.1017/sjp.2017.46, The Spanish Journal of Psychology, 20, eXX, (2017).

Goodday, S. M., Rivera, D., Foran, H., King, N., Milanovic, M., Keown-Stoneman, C. D., y Duffy, A., U-Flourish university students well-being and academic success longitudinal study: a study protocol. https://doi.org/10.1136/bmjopen-2019029854, BMJ Open, 9(8), e029854, (2019).

Hinrichs, C.P., Ortiz, L. E., y Pérez, C. E., Relación entre el Bienestar Académico de Estudiantes de Kinesiología de una Universidad Tradicional de Chile y su Percepción del Ambiente Educacional, https://dx.doi.org/10.4067/S071850062016000100012, Formación universitaria, 9(1), 109-116, (2016).

Iordache-Platis, M., Strategy for Well-Being in Universities: A Romanian Higher Education Approach. Sustainability, 12(19), 1-17, (2020).

Lugosi, P., Campus foodservice experiences and student wellbeing: An integrative review for design and service interventions, https://doi.org/10.1016/j.jijm.2018.10.019, International Journal of Hospitality Management, 83, 229-235, (2019).

Mayol, M. H., Scott, B. M., y Schreiber, J. B., Validation and Use of the Multidimensional Wellness Inventory in Collegiate Student-Athletes and First-Generation Students. https://doi.org/10.1080/19325037.2017.1345669, American Journal of Health Education, 48(5), 338-350, (2017).

MEN,, Lineamientos de política de bienestar para instituciones de educación superior, Ministerio de Educación Nacional, Bogotá D.C., Colombia, (2016).

MEN., Decreto 1330 de 2019, Ministerio de Educación Nacional, Bogotá D.C., Colombia, (2019)

CESU., Acuerdo 02 de 2020, Consejo de Educación Superior, Bogotá D.C., Colombia, (2020)

Morris-Paxton, A. A., Van Lingen, J. M., y Elkonin, D., An evaluation of health information and wellness priorities among socioeconomically disadvantaged students, https://doi.org/10.1177/0017896916670690, Health Education Journal, 76(3), 271-281, (2017).

Motefakker, N., The Study of the Level of Satisfaction of the Students of the Faculty of Social Sciences with Welfare Services of Imam Khomeini International University of Qazvin, https://doi.org/10.1016/S2212-5671(16)30052-1, Procedia Economics and Finance, 36, 399-407, (2016).

McNeilly, N., y Raming, B., Evaluation of College Student Food Safety Knowledge and Expectations of Food Service Inspections in North Carolina. Journal of Environmental Health 81 (1), 16-20, (2018).

Salcedo, V., Quezada, C., Novillo, E., Varela, G., Nuñez, L., y Viteri, C., Bienestar estudiantil universitario en Ecuador: Caso unidades de bienestar estudiantil en las universidades de la provincia de El Oro. Revista ESPACIOS, 38(30), 17, (2017).

Walker, M., The well-being of South African university students from low-income households. Oxford Development Studies, 48(1), 56-69, (2020). 